\title{
聚合物合成设计问题的教学 切断法及逆向思维在高分子化学中的应用
}

郭霖*

青岛大学材料科学与工程学院, 山东青岛 266071

摘要: 将切断法引入高分子化学教学解决聚合物合成设计问题, 并对各种常见类型聚合物的合成问题进行了分类, 提出了切断方法、切断策略, 总结了切断规律, 解决了学生面对此类问题时所普遍存在的理不出头绪、找不到思路、 想不出方法、不知该如何思考的问题, 可使相关问题变得不再棘手和相对容易解决, 并使学生不仅学会知识, 更学 会方法、具备能力。还结合切断规律, 将聚合物分类为通过官能团之间的反应进行的、通过打开不饱和键进行的, 和通过打开环进行的三种类型。将聚合物合成中的切断规律简单总结为: 切断通常是在主链上的单元之间、主链与 侧基之间、主链与侧链之间, 以及主链与端基之间, 且通常是在官能团或杂原子所在位置。

关键词: 高分子化学; 教学; 聚合物合成设计; 切断法; 聚合反应分类; 逆向思维; 知识迁移 中图分类号: G64; O63

\section{Teaching of Polymer Synthesis Design: Application of Reverse Thinking and the Disconnection Approach in Polymer Chemistry}

Lin Guo *

College of Materials Science and Engineering, Qingdao University, Qingdao 266071, Shandong Province, China.

\begin{abstract}
The disconnection approach is applied to teaching of polymer chemistry. The disconnection strategy for usual polymer synthesis problem is proposed, the rules are summarized, and the usual polymer synthesis problem is categorized. These approaches made it easier for the students to handle polymer synthesis problems, to learn the method and have the ability to learn. Accordingly, a new classification of polymerization reactions is suggested, which classifies polymerization reactions into three categories by occurring between functional groups, via opening of double bonds, and by opening of rings. It is also summarized that the disconnections in polymer synthesis are usually made between the units along the main chain, between the main chain and the side groups, between the main chain and the branches, and between the main chain and the end groups, and at the position of the functional groups or the heteroatoms.
\end{abstract}

Key Words: Polymer chemistry; Teaching; Polymer synthesis design; Disconnection approach;

Classification of polymerization reaction; Reverse thinking; Knowledge transfer

聚合物的合成, 既是高分子化学的主要任务, 也是高分子化学教学中的重要问题, 更是常常令 学生感到困难的一类问题。

收稿: 2021-01-04; 录用: 2021-03-03; 网络发表: 2021-03-26

“通讯作者, Email: guolin@qdu.edu.cn

基金资助：山东省普通高等学校教学改革试点课程 “高分子化学” 项目(鲁教高字[1999]27 号); 山东省省级精品课程 “高分子化学” 项目 (150410030); 山东省一流课程 “高分子化学” 暨青岛大学 “以学为中心” 课程 “高分子化学” 项目(RC1900004947); 青岛大学研究生教育质量 提升计划“计算机在化学中的应用”项目(RC19000016633) 
对于给定单体及聚合条件, 或给定起始聚合物及反应条件, 要求写出聚合或反应产物(目标聚合 物)结构这样一类需要正向思维的合成问题, 学生往往还比较容易找到思路和答案, 而对于已知聚合 物(即目标聚合物)结构, 要求找到合适的起始物(单体或起始聚合物)、给出正确的或各种可能的合成 路线这样一类需要逆向思维的合成设计问题, 特别是, 当目标聚合物的结构比较复杂, 或无法从其 结构一眼看出单体来源 ${ }^{[1]}$ 时, 学生们常常会感到一筹莫展, 不知该如何思考。且对于此类问题, 教科 书及教辅书中通常又往往只是简单给出答案, 并不做更多讲解, 即便是有讲解, 也往往是仅限于对 已有合成路线的介绍(从而能让学生 “知其然”), 很少介绍为什么是这样(从而能让学生 “知其所以 然” ), 而对于解决此类问题的思路、方法, 即如何才能找到这种合成方法和设计出这种合成路线, 如何才能知道应该这样来合成 (从而能让学生知道并做到 “何以知其然” ${ }^{[2]}$ ), 以及如何才能知道为什 么应该这样来合成(从而能让学生知道并做到 “何以知其所以然” ${ }^{[2]}$ )的问题, 通常都没有任何讲解。 学生也因而无法从中真正学到相关的设计方法、策略及思路, 掌握聚合物合成路线设计的方法、知 道该如何设计合成路线, 并有能力自己设计合成, 当然, 其创造力和创造思维也就无法很好地得到 培养。

因此, 在高分子化学教学中, 任课教师如何能从根本上解决这个问题, 使学生不仅能有聚合物 合成相关的知识, 而且能有解决聚合物合成设计问题的思路、方法、能力, 不仅仅只是知道、理解 或掌握已有或已知的聚合物合成路线, 而是能在面对未知合成路线的聚合物合成问题时, 设计出合 适的聚合物合成路线, 就显得尤为重要, 也是摆在所有高分子化学课程任课教师面前的一项艰巨的 任务和挑战。

由于高分子化学课程中, 通常讨论的都是有机高分子或元素有机高分子, 它们都可视作是一类 特殊的有机物——类分子量非常高, 且分子量往往还有分布, 结构也通常比较复杂, 但却又会有 重复特征的有机物(当然, 也有人 ${ }^{[3]}$ 将元素有机高分子视作无机高分子), 因此, 其合成问题自然可以 视作有机合成问题, 其合成设计当然就可以沿用有机合成中常用的方法, 即, 可以将聚合物合成设 计问题转换成或视作有机合成设计问题，并采用有机合成设计的方法来进行合成设计。

切断法, 或合成子法, 是一种从目标化合物开始, 通过 “切断” 和倒推, 直至简单起始原料(或 称合成等价物)的逆向分析法或反合成分析法 ${ }^{[4,5]}$, 是一种在有机化学教学中已被广为介绍 ${ }^{[6]}$, 且行之 有效的有机合成设计方法, 特别是对于结构比较复杂的有机物的合成更是如此。

根据上述分析不难推测, 若将其用于聚合物合成设计也应行之有效。

然而, 令人遗憾的是, 迄今尚未见高分子化学教科书、教辅书及高分子化学教学中有类似尝试 或相关介绍。

为此, 我们进行了相关探索, 尝试将切断法用于聚合物合成设计及聚合物合成设计教学, 在帮 助学生解决相关问题的同时, 培养学生的逆向思维能力及知识迁移能力, 并取得良好效果。

\section{1 聚合反应的分类方法}

通常, 聚合反应可以按照Carothers的方法分类, 分为加聚、缩聚、开环聚合、聚加成、加缩聚、 Diels-Alder聚合、氧化偶合聚合等; 可以按照Flory的方法分类, 分为连锁聚合、逐步聚合。

我们根据聚合所对应的反应类型、反应特征、单体结构、反应部位的不同(因而所得聚合物主 链结构、结构特征不同, 切断规律, 包括切断方式及切断后所得合成等价物, 也就是单体或起始聚 合物，也会不同)，将聚合反应分为如下三类。

\section{1 通过官能团之间的反应进行的聚合}

此类聚合包括Carothers分类体系中的缩聚、聚加成、加缩聚、Diels-Alder聚合、氧化偶合聚合等， 对应于Flory分类体系中的逐步聚合。

由于官能团多涉及C原子之外的原子, 因此, 此类聚合反应得到或涉及的聚合物通常均是杂链聚 合物或元素有机聚合物, Diels-Alder聚合及其所得聚合物是其中为数不多的例外。 


\section{2 通过打开不饱和键进行的聚合}

此类聚合对应于Carothers分类体系中的加聚, 包括阳离子聚合中的异构化聚合、阴离子聚合中 的氢转移聚合(如, 丙烯酰胺的阴离子聚合), 以及环烯烃的(得到不开环的环烯烃聚合产物的)双键聚 合(加成聚合)等, 对应于Flory分类体系中的连锁聚合。

这里的不饱和键主要是 $-\mathrm{C}=\mathrm{C}-$ 及 $-\mathrm{C}=\mathrm{O} 、-\mathrm{C} \equiv \mathrm{C}-$ 。

由于此类聚合中通常主要是 $-\mathrm{C}=\mathrm{C}$-的聚合, 因而, 得到或涉及的聚合物一般都是碳链聚合物。 只有像丙烯酰胺的阴离子聚合等, 才会涉及杂链聚合物。

\section{3 通过打开环进行的聚合}

此类聚合对应于Carothers分类体系中的开环聚合、开环易位聚合等。按照Flory的分类体系, 此 类聚合主要是连锁聚合, 也有部分为逐步聚合。

由于此类聚合中通常打开的都是杂环, 因而, 得到或涉及的聚合物主要是杂链聚合物或元素有 机聚合物, 只有在开环易位聚合中才会涉及碳链聚合物。

\section{2 聚合物合成的基本方法}

聚合物的合成路线或方法通常有两种, 一种是从单体出发, 通过单体聚合直接得到, 即, $M \rightarrow P$ (合成方法 I, 与上述1.1-1.3节三种聚合反应相对应的合成方法可分别表示为合成方法I-1、合成方法 I-2 以及合成方法I-3); 另一种是从另一种聚合物(起始聚合物)出发, 通过聚合物的化学反应得到, 即, $\mathrm{P}^{\prime} \rightarrow \mathrm{P}$ (合成方法 $\mathrm{II}$ )。

结构不同的聚合物，其合成方法虽有不同，但有规律。

杂链聚合物、元素有机聚合物, 以及绝大多数碳链聚合物, 包括均聚物、无规共聚物、交替共 聚物等, 通常通过合成方法I合成。其中杂链聚合物、元素有机聚合物一般通过合成方法I-1或/和合 成方法I-3合成, 碳链聚合物则一般通过合成方法I-2合成。

少部分碳链聚合物, 如聚乙烯醇等, 采用合成方法II合成。

而遥爪预聚物、大单体、嵌段共聚物、接枝共聚物等, 则往往采取合成方法I与合成方法II联用 的方法合成。有些碳链聚合物, 如氯化聚乙烯等, 也采用此种方法合成。

\section{3 聚合物合成设计中的切断方法、切断策略与切断规律}

聚合物合成中的切断, 主要涉及主链上的单元之间、主链与端基之间、主链与侧基之间(包括主 链上的 $\mathrm{C}-\mathrm{H}$ 之间), 以及主链与侧链之间四种不同方式的切断。其中, 主链上单元之间的切断(切断 方式(1))可以归为一类, 主链与端基之间(切断方式(2)、主链与侧基之间(切断方式(3), 以及主链与侧 链之间(切断方式(4))的切断, 则可归为另外一类。并可认为, 两类不同的切断, 分别对应两种不同的 切断策略, 或两种合成聚合物的不同方法, 前者对应于各种聚合反应(合成方法I), 后者则对应于各 种聚合物的化学反应(合成方法II)。

结构不同的聚合物，合成方法不同，作为其合成过程逆过程的切断，当然也就不同。

拿到目标聚合物的结构以后, 首先应该分析判断其是否是(或可否)通过单体聚合直接得到, 如果 是的话, 再具体判断是或者可以通过哪一类聚合得到, 然后, 再进行相应的切断。条件允许以及必 要的情况下, 还可进一步, 结合Scifinder ${ }^{\mathrm{n}}$ 中的逆合成检索加以验证。

无论进行哪一种切断, 其前提都是要正确地找出并确定目标聚合物的(重复单元的)结构, 具有进 行切断所必须的有机化学基础知识, 以及足够的观察能力—从一而能够选择和确定符合反应规律的、 合适的切断方式(对应于选择上述哪一种方法或路线来合成聚合物), 并找出合适的切断点及具体切 断方法。

\section{1 通过官能团之间的反应进行的聚合所得聚合物的切断}

首先要判断目标聚合物是否属于, 或可否通过此类聚合得到, 此类聚合所得聚合物的特征通常 
是, 杂链聚合物或元素有机聚合物。当然, 具有此类特征的聚合物也可能是通过打开环进行的聚合, 亦即开环聚合得到的。

对于按此类聚合所得到的目标聚合物, 通常是在聚合物主链的单元间, 包括重复单元之间以及 重复单元中的各结构单元之间, 进行切断, 切断位置应该在官能团或杂原子处, 具体方式或所得合 成子, 则需要考虑具体结构, 特别是官能团结构。具体切断方法与有机化学中相同, 因为, 此时可 将聚合物(重复单元)视作普通的有机物。

除此之外, 切断前还要判定其一个重复单元是否由两个结构单元构成, 如果是的话, 则要首先 在两个结构单元的连接处切断。

\section{2 通过打开不饱和键进行的聚合所得聚合物的切断}

首先, 也要判断目标聚合物是否属于, 或可否通过此类聚合得到, 此类聚合所得聚合物的特征 是, 通常皆为碳链聚合物, 只有像丙烯酰胺的阴离子聚合等所得的聚合物, 才会涉及杂链聚合物。

对于按此类聚合所得到的目标聚合物, 通常是在聚合物主链的单元间, 包括重复单元之间以及 重复单元中的各结构单元之间, 也就是主链上相邻两个单元间相连的 $-\mathrm{C}-\mathrm{C}-$ 处, 进行切断, 得到 对应的烯类单体。

需要注意的是, 如果切断所得合成等价物, 即预期中的单体, 并不存在, 如乙烯醇(聚乙烯醇按 此方法切断时所得的合成等价物), 或者还含有另外一个可能干扰聚合的双键(即目标聚合物为侧基 中含有 $-\mathrm{C}=\mathrm{C}$-的碳链聚合物时), 如肉桂酸乙烯酯(聚肉桂酸乙烯酯按此方法切断所得的合成等价 物)等, 或者位阻太大, 如对甲苯丙烯酸甲酯等, 则都不能按此方法切断。此时, 须视具体情况(聚合 物结构), 考虑从侧基处或按其他方式切断, 即考虑通过聚合物的化学反应(合成方法II)来合成, 而非 直接通过单体的聚合(合成方法I) 来合成。经常有人会在此问题上犯错 ${ }^{[7,8]}$, 如, 有人错误地认为存在 高聚物聚对甲苯丙烯酸甲酯, 并想当然地认为其合成等价物, 即其单体, 为对甲苯丙烯酸甲酯 ${ }^{[7]}$, 但 实际情况却是, 该聚合物并不存在, 或更严格地讲, 该聚合物迄今尚无人合成出, 关于这一点, 我 们在前期工作 ${ }^{[9]}$ 中已进行了专门介绍, 这里不再赘述。

总而言之, 此类聚合物切断时, 既要考虑切断所得合成等价物, 即预期中的单体, 是否真实存 在, 也要考虑预期中的单体中是否含有另外一个 $-\mathrm{C}=\mathrm{C}-$, 以及含有的另外这个 $-\mathrm{C}=\mathrm{C}-$ 是否会在 给定的聚合条件下参与或干扰聚合, 还要考虑预期中的单体是否真的能够(均)聚合, 或真的不会因位 阻太大而无法(均)聚合, 如对甲苯丙烯酸甲酯等 ${ }^{[7,8]}$ 位阻非常大的 1,2 -二取代的烯类化合物。

此外, 切断之前, 还要先判断目标聚合物的重复单元是否可以分解为两个结构单元, 或者其两 个结构单元是否可以合并为一个更大的结构单元或不能再分的重复单元, 并避免在这两个单元之间 进行不必要的切断。

\section{3 通过打开环进行的聚合所得聚合物的切断}

首先, 还是要判断目标聚合物是否属于, 或者可以通过此类聚合得到, 此类聚合所得聚合物与 通过官能团间的反应制备的聚合物常常具有相同的结构特征, 甚至有些聚合物也的确可以分别通过 这两种不同的聚合方法得到, 如聚己内酰胺、聚环氧乙烷、聚乳酸等。

此类聚合所得聚合物的特征, 也是为杂链聚合物, 或元素有机聚合物, 但开环易位聚合所得聚 合物是碳链聚合物, 是个例外。

此类聚合所得聚合物的切断方法, 与上述 3.1 节中的类似。

\section{4 通过聚合物的化学反应合成的聚合物的切断}

首先, 须判断目标聚合物需采用或可以采用此种方法进行合成, 然后, 根据前面介绍的切断方 法进行切断。

此类聚合物的切断通常是在主链与侧基之间(包括主链上的 $\mathrm{C}-\mathrm{H}$ 之间)、主链与侧链之间, 以及 主链与端基之间。 


\section{5 聚合物合成设计中的切断策略与切断规律}

聚合物合成设计中的切断策略与切断规律可总结如图1所示, 相关说明见表 1 (具体例子见第4节)。

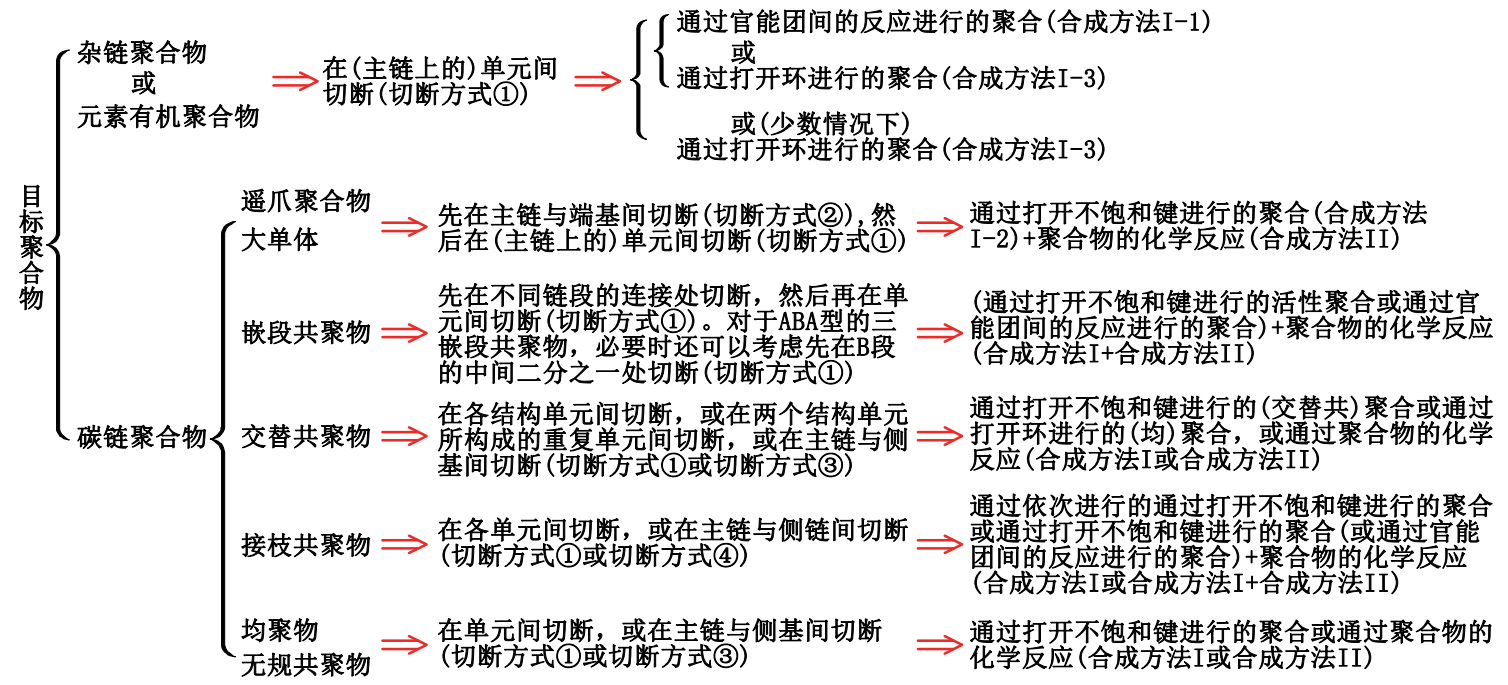

图1 各种类型聚合物的切断方式与合成方法

表1 不同种类聚合物的判断方法、切断方式及合成方法

\begin{tabular}{|c|c|c|c|c|}
\hline 聚合物类型 & 判断方法 & 切断方式 & 合成方法 & 举例编号 \\
\hline 碳链聚合物 & 聚合物主链中只含C & $\begin{array}{c}\text { 切断方式(1) } \\
\text { 或 } \\
\text { (切断方式(3) } \\
+ \\
+ \\
\text { 切断方式(1) }\end{array}$ & $\begin{array}{c}\text { 合成方法I-2 } \\
\text { 或 } \\
\text { (合成方法I-2 } \\
\quad+ \\
\text { 合成方法II) }\end{array}$ & $\begin{array}{c}6,7,8,11,12,13, \\
14,15,16,18,20 \\
17 \text { (尚未实现) }\end{array}$ \\
\hline 杂链聚合物 & 聚合物主链中不仅含 $\mathrm{C}$, 还含有 $\mathrm{C}$ 之外的原子, 如 $\mathrm{O} 、 \mathrm{~N}$ 等 & \multirow[t]{2}{*}{ 切断方式(1) } & \multirow[b]{2}{*}{$\begin{array}{l}\text { (合成方法I-1 } \\
\text { 或/和 } \\
\text { 合成方法I-3) } \\
\text { 或 } \\
\text { 少数情况下 } \\
\text { 合成方法I-2 }\end{array}$} & \multirow{2}{*}{$\begin{array}{c}1,2,3,4,5,9,10, \\
19\end{array}$} \\
\hline 元素有机聚合物 & 聚合物主链中不含 C, 但侧基中含 C & & & \\
\hline 遥爪聚合物 & $\begin{array}{l}\text { 聚合物链端含有反应性基团。但诸如聚酰胺、聚酯等缩聚物, } \\
\text { 虽然链端有 }-\mathrm{COOH} 、-\mathrm{NH}_{2} 、-\mathrm{OH} \text { 等反应性基团, 通常却 } \\
\text { 不被认为是遥爪聚合物 }\end{array}$ & \multirow[t]{2}{*}{$\begin{array}{c}\text { 切断方式(2) } \\
+ \\
+ \\
\text { 切断方式(1) }\end{array}$} & \multirow[t]{2}{*}{$\begin{array}{l}\text { 合成方法I-2 } \\
+ \\
+ \\
\text { 合成方法II }\end{array}$} & \multirow[t]{2}{*}{7,8} \\
\hline 大单体 & $\begin{array}{l}\text { 聚合物链端含有聚合性基团。有些基团, 如环氧基, 在不同 } \\
\text { 反应条件下, 可以分别是反应性基团或聚合性基团 }\end{array}$ & & & \\
\hline 嵌段共聚物 & $\begin{array}{l}\text { 聚合物(主)链中至少含有两段由不同单体构成的较长的链 } \\
\text { 段或序列 }\end{array}$ & $\begin{array}{c}\text { 切断方式(1) } \\
\text { 或 } \\
\text { (切断方式(2) } \\
+ \\
+ \\
\text { 切断方式(1) }\end{array}$ & $\begin{array}{c}\text { 合成方法I } \\
\text { 或 } \\
\text { (合成方法I } \\
\quad+ \\
\text { 合成方法II) }\end{array}$ & 6 \\
\hline
\end{tabular}


大学化学 Univ. Chem. 2021, 36 (12), 2101004 (6 of 11)

(续表1)

\begin{tabular}{|c|c|c|c|c|}
\hline 聚合物类型 & 判断方法 & 切断方式 & 合成方法 & 举例编号 \\
\hline 交替共聚物 & 聚合物链由两种来自不同单体的单元交替连接而成 & $\begin{array}{l}\text { 切断方式(1) } \\
\text { 或 } \\
\text { 切断方式(3) }\end{array}$ & $\begin{array}{l}\text { 合成方法I } \\
\text { 或 } \\
\text { 合成方法II }\end{array}$ & $9,10,11$ \\
\hline 接枝共聚物 & $\begin{array}{l}\text { 聚合物分子中有一条贯穿整个分子的长链 (主链)和若干连 } \\
\text { 接在主链上的、由来自其他单体的单元所构成的短链(支链) }\end{array}$ & $\begin{array}{l}\text { 切断方式(1) } \\
\text { 切断方式(3) } \\
\text { 切断方式(4) }\end{array}$ & $\begin{array}{c}\text { 合成方法I } \\
\text { 或 } \\
\text { (合成方法I } \\
+ \\
+ \\
\text { 合成方法II) }\end{array}$ & $\begin{array}{l}\text { 高分子化学教科书 } \\
\text { 中对此类聚合物的 } \\
\text { 合成有比较充分的 } \\
\text { 讲解, 本文未举例 }\end{array}$ \\
\hline 无规共聚物 & $\begin{array}{l}\text { 聚合物链至少由两种来自不同单体的单元随机相连而成, 通 } \\
\text { 常为碳链聚合物的, 也可为杂链聚合物 (如通过打开环进行 } \\
\text { 的聚合或通过官能团之间的反应进行的聚合所得的共聚物) }\end{array}$ & $\begin{array}{l}\text { 切断方式(1) } \\
\text { 切断方式(3) }\end{array}$ & $\begin{array}{l}\text { 合成方法I-2 } \\
\text { 或 } \\
\text { 合成方法I-3 }\end{array}$ & 20 \\
\hline 均聚物 & $\begin{array}{l}\text { 聚合物链由来自同一种单体的单元(可以不只一种)相连而 } \\
\text { 成, 通常为碳链聚合物的, 也可为杂链聚合物(如开环聚合 } \\
\text { 物) }\end{array}$ & & $\begin{array}{c}\text { 或 } \\
\text { 合成方法I-2 }\end{array}$ & $\begin{array}{c}1,2,9,12,13,14 \\
18,19\end{array}$ \\
\hline
\end{tabular}

\section{4 切断法进行聚合物合成设计应用举例}

我们选取了高分子化学课程中的部分典型的聚合物合成设计问题, 作为用切断法进行聚合物合 成设计的应用举例, 列于表2。

表2 切断法聚合物合成设计应用举例

\begin{tabular}{|c|c|c|c|}
\hline 编号 & 举例 & 切断方式 & 合成方法 \\
\hline 1 & 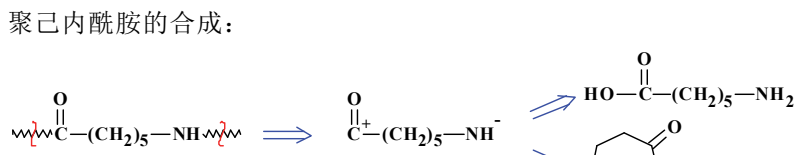 & 切断方式 (1) & $\begin{array}{l}\text { 合成方法I-1 } \\
\text { 或/和 } \\
\text { 合成方法I-3 }\end{array}$ \\
\hline 2 & 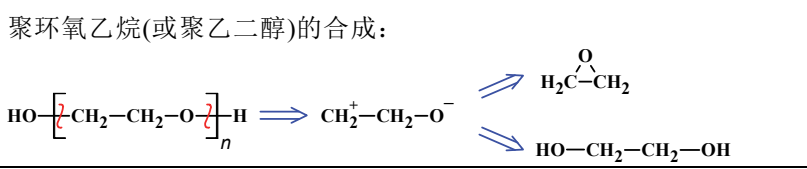 & & \\
\hline 3 & 聚对蓝二甲酸乙二酯(PET)的合成: & & \\
\hline 4 & 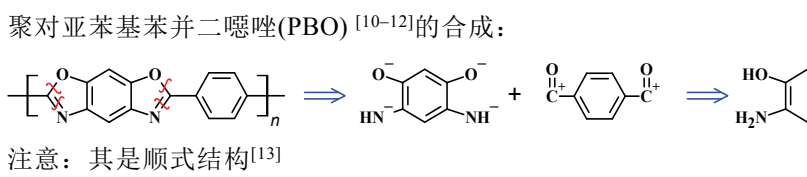 & & 合成方法I-1 \\
\hline
\end{tabular}




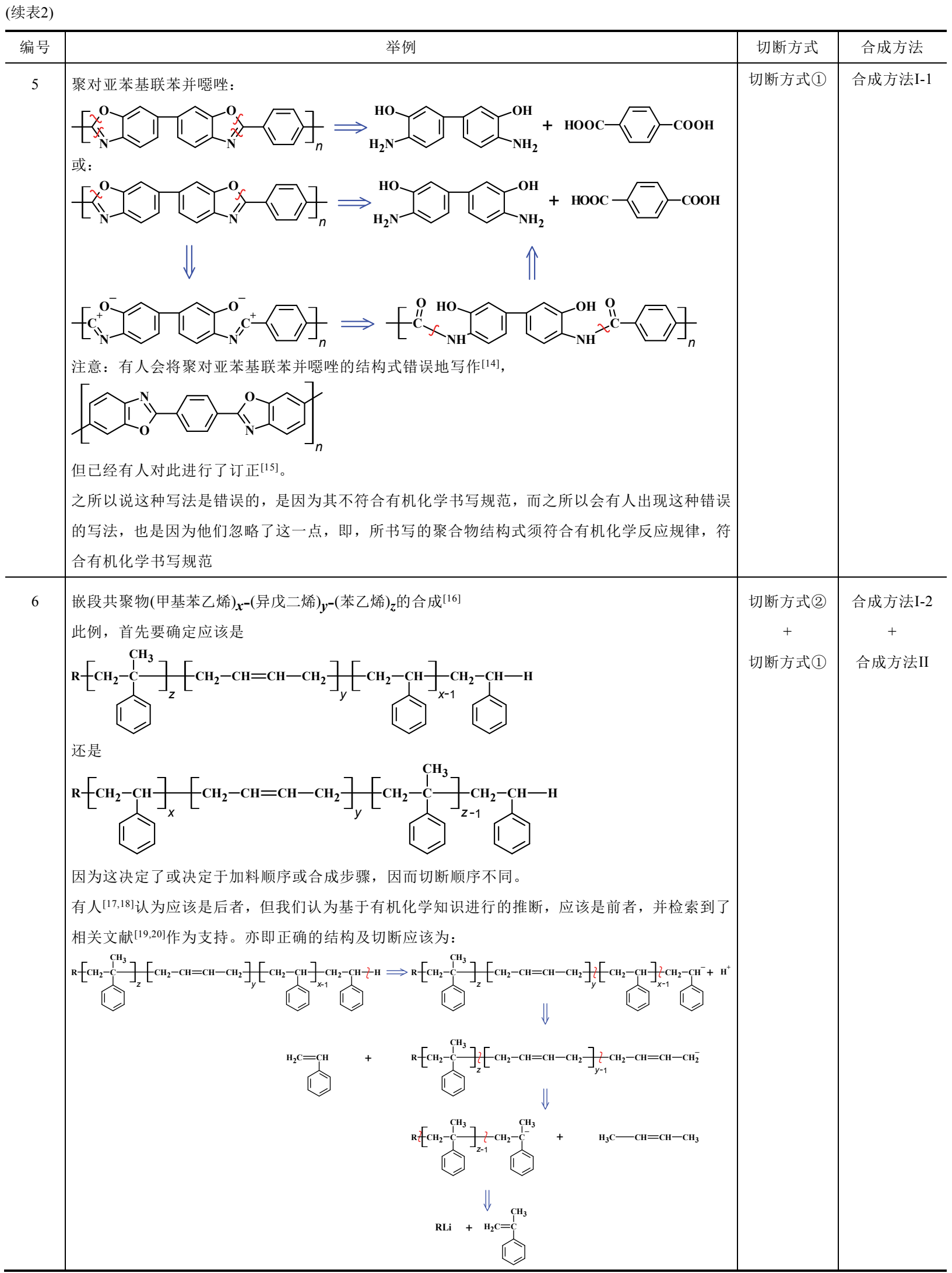




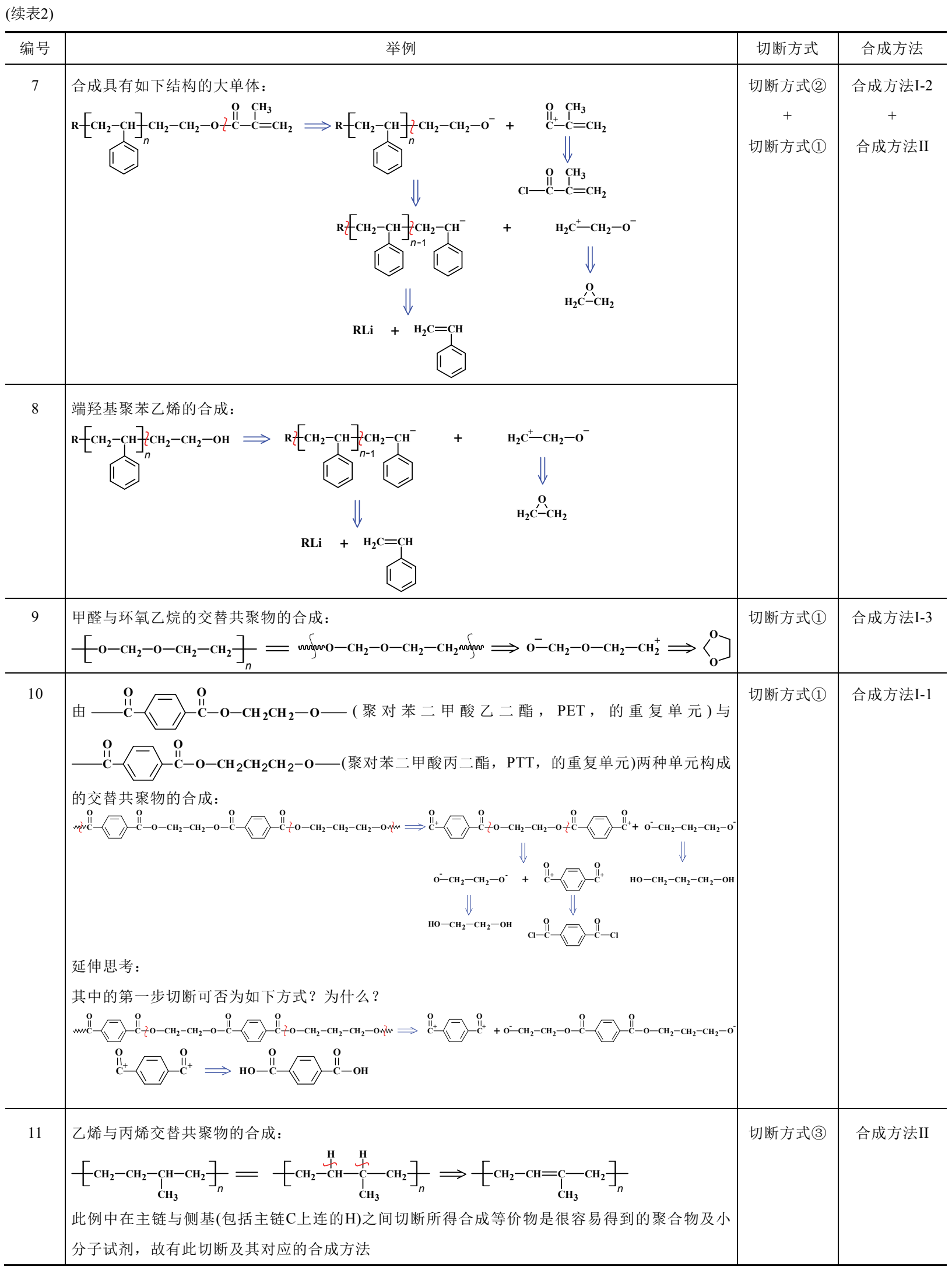




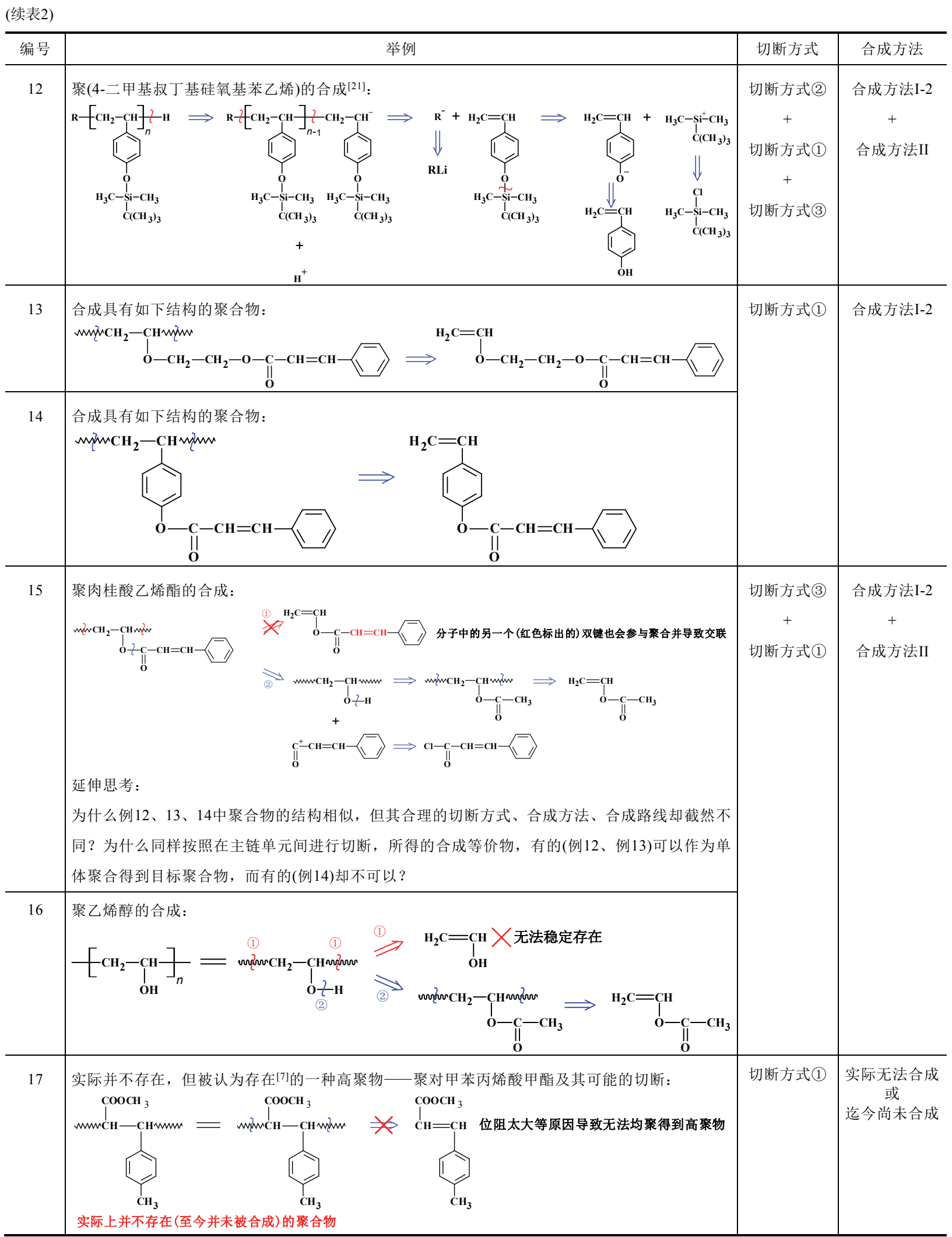




\begin{tabular}{|c|c|c|c|}
\hline 编号 & 举例 & 切断方式 & 合成方法 \\
\hline 18 & 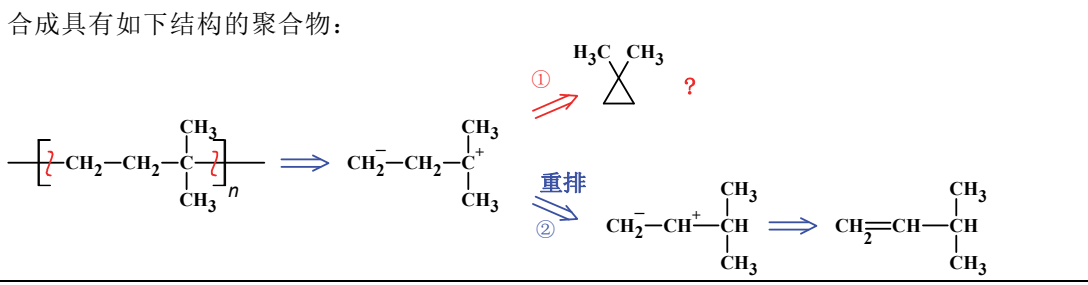 & $\begin{array}{l}\text { 切断方式(1) } \\
+ \\
\text { 重排(的逆过 } \\
\text { 程) }\end{array}$ & 合成方法 I-2 \\
\hline 19 & 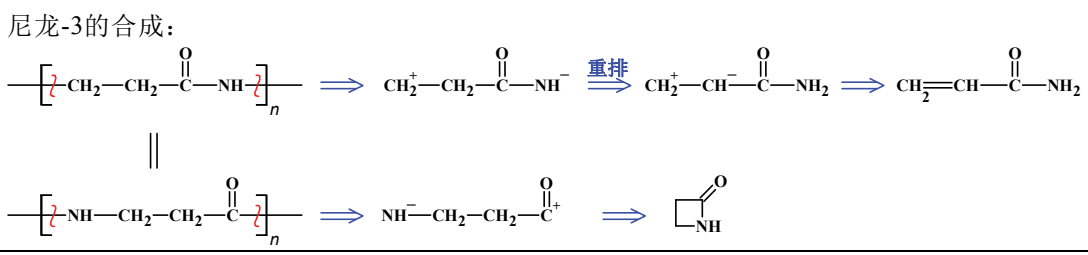 & $\begin{array}{c}\text { 切断方式(1) } \\
+ \\
\text { 重排 } \\
\text { 或 } \\
\text { 切断方式(1) }\end{array}$ & $\begin{array}{l}\text { 合成方法 } \mathrm{I}-2 \\
\text { 或 } \\
\text { 合成方法I-3 }\end{array}$ \\
\hline 20 & 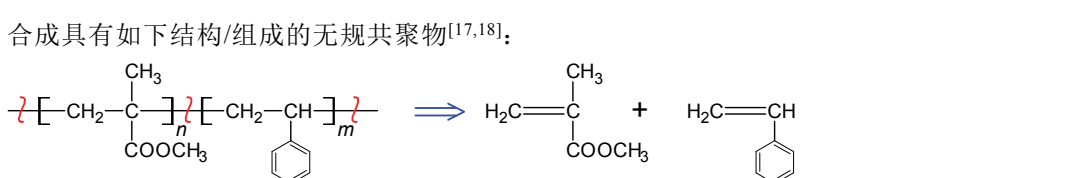 & 切断方式 (1) & 合成方法I-2 \\
\hline
\end{tabular}

\section{5 结语}

切断法是有机化学中进行合成设计时常用的方法, 将其用于聚合物合成设计同样行之有效。该 方法可使聚合物合成设计问题变得不再棘手和相对容易解决, 且能帮助学生在学会知识的同时, 学 会方法、学会思考、具备能力, 既能使当前问题迎刃而解, 举重若轻, 又能帮助学生学会举一反三、 做到触类旁通, 可谓一举多得。

提出的聚合反应新分类方法, 则可使学生更容易地理解和掌握聚合物合成设计时的切断方法与 切断规律。

\section{参 考 文 献}

[1] 林权, 崔占臣. 高分子化学. 北京: 高等教育出版社, 2015: 7.

[2] 郭霖, 隋坤艳, 丛海林, 张小艳, 王久兴, 王彦欣. 大学化学, 2021, 36 (4), 2005073.

[3] 西久保忠臣. 高分子化学. 范星河, 谢晓峰, 译. 李子臣, 校译. 北京: 北京大学出版社, 2013: 17.

[4] 斯图尔特.沃伦. 有机合成设计(合成子法的习题解答式教程). 丁新腾, 林子森, 译. 上海: 上海科学技术文献出版社, 1981.

[5] Stuart, W.; Paul, W. Organic Synthesis: The Disconnection Approach, 2nd ed.; John Wiley \& Sons Ltd.: West Sussex, UK, 2008.

[6] 徐寿昌. 有机化学. 北京: 高等教育出版社, 1993: 487.

[7] a) 2014年山东省高考理综卷34题及其标准答案. [2020-12-02]. http://sd.dzwww.com/sdnews/201406/t20140608_10413279.htm b) 2014年高考(山东卷)化学试题评析. [2021-03-25]. http://www.huaue.com/gkao2014/201468194206.htm c) 2014年山东高考化学试题评析: 突出信息整合能力 实验探究能力考查. [2021-03-25].

http://www.sdnews.com.cn/sd/yw/201406/t20140608_1633055.htm

[8] 王后雄. 高考完全解读. 化学: 课标版. 北京: 中国青年出版社, 2006: 195.

[9] 郭霖, 隋坤艳, 胡浩, 王久兴, 王逸凡. 张小艳, 彭乔虹, 随欣. 大学化学, 2021, 36 (8), 2009004

[10] 雷晖, 冯东东, 袁明祥, 李欣欣, 庄启昕, 吴平平, 韩哲文. 功能高分子学报, 2003, $16(1), 120$.

[11] 袁江, 王建营, 胡文祥, 孙家跃. 化工纵横, 2003, 17 (8), 4.

[12] 李金焕, 徐辉, 黄玉东. 合成树脂与塑料, 2003, $20(6), 16$. 
[13] 金宁人, 刘斌, 胡建明, 孙建庭, 赵德明. 化工进展, 2010, 29 (8), 1547.

[14] 吴国庆. 化学教育, 2001, No. 1, 40.

[15] 魏荣宝. 化学教育, 2001, No. 3, 47.

[16] 潘祖仁. 高分子化学. 第5版. 北京: 化学工业出版社, 2011: 191.

[17] 贾红兵. 高分子化学导读与题解. 第5版. 北京: 化学工业出版社, 2013: 125 .

[18] 贾红兵. 高分子化学导读与题解. 第4版. 北京: 化学工业出版社, 2009: 125.

[19] Hsieh, H. C.; Kao, H. C.;Cheng, D. O.; Tsiang, R. C.; Huang, D. Macromolecules 1995, $28,4383$.

[20] Yamashita, Y.; Hane, T. J. Polym. Sci.: Part A: Polym. Chem. 1973, 11, 425.

[21] 唐黎明, 度新林. 高分子化学. 第2版. 北京: 清华大学出版社, 2016: 187. 